\title{
White matter microstructure and volume correlates of premenstrual dysphoric disorder
}

\author{
Xuan Gu, PhD*; Manon Dubol, PhD*; Louise Stiernman, MD; Johan Wikström, PhD; \\ Andreas Hahn, PhD; Rupert Lanzenberger, MD, PD; C. Neill Epperson, PhD; \\ Marie Bixo, PhD; Inger Sundström-Poromaa, $\mathrm{PhD}^{\dagger}$; Erika Comasco, $\mathrm{PhD}^{+}$
}

\begin{abstract}
Background: Premenstrual dysphoric disorder (PMDD) is a mood disorder characterized by psychological and physical symptoms. Differences in white matter have been associated with affective and anxiety disorders, which share some symptoms with PMDD. However, whether white matter structure differs between the brains of individuals with PMDD and healthy controls is not known, nor is its relation to symptom severity. Methods: We performed tract-based spatial statistics and voxel-based morphometry analyses of diffusion tensor imaging metrics and white matter volume, using 2 neuroimaging data sets ( $n=67$ and $n=131$ ) and a combined whole-brain and regionof-interest approach. We performed correlation analyses to investigate the relationship between regions with different white matter microstructure and volume and PMDD symptom severity. Results: We found greater fractional anisotropy in the left uncinate fasciculus $(d=0.69)$ in individuals with PMDD compared to controls. Moreover, the volume of the right uncinate fasciculus was higher in individuals with PMDD compared to controls $(d=0.40)$. As well, the severity of premenstrual depression was positively correlated with fractional anisotropy in the right superior longitudinal fasciculus $(r=0.35)$. Limitations: It is challenging to interpret group differences in diffusion tensor imaging metrics in terms of their underlying biophysical properties. The small size of the control group in the diffusion tensor imaging study may have prevented effects of interest from being detected. Conclusion: The findings of the present study provide evidence of differential cerebral white matter structure associated with PMDD and its symptoms.
\end{abstract}

\section{Introduction}

Premenstrual dysphoric disorder (PMDD) is a mood disorder that affects about $3 \%-8 \%$ of individuals who menstruate. ${ }^{1}$ It is characterized by symptoms of affective lability, irritability, depression and anxiety, peaking in the late luteal phase of the menstrual cycle. ${ }^{2}$

Since its recognition as a distinct psychiatric disorder in the DSM $-5,{ }^{3}$ the etiology of PMDD has become an increasingly active area of research. In the search for the neural correlates of PMDD, MRI has been employed to investigate brain structure and function in individuals with PMDD. ${ }^{4}$ Notably, functional imaging studies have suggested an impairment of topdown inhibitory circuits that comprise the corticolimbic brain networks, ${ }^{4}$ likely involving altered connections between the frontal and subcortical regions. Nevertheless, whether there are structural alterations in corresponding white matter tracts related to PMDD remains unexplored.
Diffusion tensor imaging (DTI) enables an assessment of white matter microstructure in vivo by measuring the diffusion of water molecules in neural fibres. ${ }^{5}$ It estimates the degree of directionality by measuring fractional anisotropy (FA), and the movement of molecules as defined by mean diffusivity (MD), axial diffusivity (AD) and radial diffusivity (RD). Alterations in anisotropy and diffusivity measures have been associated with different affective and anxiety disorders $^{6-8}$ that share symptoms with PMDD. Notably, reduced FA in the superior longitudinal fasciculus (SLF), uncinate fasciculus, inferior fronto-occipital fasciculus, forceps minor, anterior thalamic radiation, superior corona radiata, cerebellum and cingulum appears to characterize these disorders. ${ }^{6}$ Moreover, menstrual cycle phase-dependent white matter correlations with gonadal hormone levels have been described in healthy naturally cycling individuals, ${ }^{9}$ linking FA measures in the hippocampus to estradiol concentrations over the menstrual cycle, for example. ${ }^{10}$ Thus, trait-like characteristics or

Correspondence to: Erika Comasco, Department of Neuroscience, Science for Life Laboratory, Uppsala University, 75124 Uppsala,

Sweden; erika.comasco@neuro.uu.se

*These authors contributed equally to this work.

tThese authors contributed equally to this work.

Submitted Aug. 13, 2021; Revised Nov. 18, 2021; Accepted Dec. 20, 2021

Cite as: J Psychiatry Neurosci 2022 February 23;47(1). doi: 10.1503/jpn.210143 
maladaptive hormone-driven changes in white matter structure may occur in the brains of individuals with PMDD, which could relate to functional imaging findings. ${ }^{9}$

The present studies were aimed at determining whether white matter structure is altered in individuals with PMDD compared to healthy controls, using DTI data analyzed with tract-based spatial statistics (study I), and voxel-based morphometry (VBM; study II). We compared FA, diffusivity metrics (MD, $A D$ and $R D)$ and white matter volumes in individuals with PMDD and controls who were scanned during the symptomatic phase of the menstrual cycle, using a combined whole-brain and region-of-interest approach. In accordance with the neuroimaging literature on PMDD, ${ }^{4}$ as well as DTI findings related to the menstrual cycle ${ }^{9}$ and affective and anxiety disorders, ${ }^{6}$ we expected white matter structure to be altered in individuals with PMDD compared to controls. Specifically, we expected to find differences in the following white matter tracts: anterior thalamic radiation, cerebellum, cingulum, corticospinal tract, fornix, genu of the corpus callosum, inferior fronto-occipital fasciculus, inferior longitudinal fasciculus, superior corona radiata, SLF and uncinate fasciculus. We also sought to determine whether differential white matter metrics were associated with symptom severity in individuals with PMDD.

\section{Methods}

\section{Study I: white matter microstructure}

\section{Participants}

The DTI study was carried out at the Departments of Obstetrics and Gynecology at Uppsala University Hospital (site 1), between December 2016 and December 2019. In total, 62 individuals with PMDD and 11 healthy, naturally cycling individuals with regular menstrual cycles (24-35 days) were recruited using advertisements in local newspapers, local message boards, social media and student websites. Exclusion criteria were as follows: steroid hormone treatment (including hormonal contraceptives) or treatment with psychotropic drugs in the previous 3 months, breastfeeding, pregnancy, an ongoing psychiatric disorder other than PMDD, clinically relevant findings on physical examination or blood testing, and contraindications for MRI.

Study procedures complied with the Declaration of Helsinki and were approved by the ethics committee of Uppsala University (Dnr. 2016/184 and 2016/312). We obtained written informed consent from all participants after they had been given oral and written information about the study.

All participants filled in daily prospective symptom ratings over 2 consecutive menstrual cycles using the Daily Record of Severity of Problems (DRSP) scale. ${ }^{11}$ We defined a diagnosis of PMDD based on DSM-5 criteria: an increase of more than $50 \%$ in at least 5 of 11 symptoms (among them at least 1 core PMDD symptom) between the follicular phase (days 6 to 12 ) and the luteal phase (days -7 to -1$).{ }^{12}$ To meet the criteria for a diagnosis of PMDD, participants had to have marked symptoms in the luteal phase that caused substantial distress or interference with their usual activities. In ad- dition, diagnostic symptoms had be at least mild (mean luteal phase score greater than 3.0, with at least 2 days greater than 4.0) and disappear during the follicular phase (mean follicular phase score less than 2.0).

We ruled out psychiatric comorbidities using the MiniInternational Neuropsychiatric Interview. ${ }^{13}$ We also assessed and confirmed monitoring of the menstrual cycle phase using serum progesterone and estradiol concentrations measured at the same time as the brain scan. We assessed premenstrual symptom severity during the scan month using the DRSP scale. We assessed mean total DRSP scores for the late luteal phase (obtained during the final 5 days of the scan month) and the 8 items related to the core affective PMDD symptoms according to the DSM-5 (depression, hopelessness, guilt, being easily hurt, mood swings, anxiety, irritability, conflicts) ${ }^{11}$

Six participants (5 with PMDD, 1 control) were excluded from the analyses because of technical issues $(n=3)$, excessive head motion $(n=2)$ or a brain tumour $(n=1)$, for a total sample of 57 individuals with PMDD and 10 controls (Appendix 1, Figure S1, available at www.jpn.ca/lookup/ doi/10.1503/jpn.210143/tab-related-content).

\section{MRI acquisition}

All participants underwent MRI scanning, including DTI, in the luteal phase of their menstrual cycle, on a 3.0 T wholebody scanner (Achieva dStream, Philips Medical Systems) equipped with a 32-channel head coil. We acquired DTI data using a whole-brain echo planar imaging sequence with the following parameters: repetition time $3628 \mathrm{~ms}$; echo time $83 \mathrm{~ms}$; field of view $224 \mathrm{~mm} \times 224 \mathrm{~mm}$; matrix $128 \times 128$; voxel size $1.75 \times 1.75 \times 2 \mathrm{~mm}^{3}$; slice thickness $2 \mathrm{~mm}$; flip angle $90^{\circ} ; 48$ diffusion-sensitive directions with $b=$ $800 \mathrm{~mm}^{2} / \mathrm{s} ; 1$ volume with $b=0 \mathrm{~mm}^{2} / \mathrm{s} ; 60$ slices without an interslice gap; and acquisition time $7 \mathrm{~min}, 48 \mathrm{~s}$. Across participants, the mean signal-to-noise ratio in white matter was 40.3 for $b=0 \mathrm{~mm}^{2} / \mathrm{s}$ and 22.9 for $b=800 \mathrm{~mm}^{2} / \mathrm{s}$.

\section{Data preprocessing}

We performed preprocessing of the DTI data and further analyses using the FMRIB Software Library (FSL version 6.0.4; www.fmrib.ox.ac.uk/fsl/index.html) and the default recommended parameters. We corrected for head motion and eddy current distortions by registering all volumes to the first volume using the FSL function eddy_correct. ${ }^{14,15}$ We used the FSL function $\mathrm{BET}^{16}(f=0.4, g=0)$ to create the brain mask from the $b_{0}$ image. We performed diffusion tensor fitting and DTI metric (FA, MD, AD) calculations using the FSL function dtifit. We performed RD calculation using the FSL function fslmaths, with $\operatorname{RD}=\left(\lambda_{2}+\lambda_{3}\right) / 2$, where $\lambda_{2}$ and $\lambda_{3}$ were the 2 smaller eigenvalues. After a visual quality check, we excluded 2 participants from subsequent analyses because of severe head motion.

Tract-based spatial statistics allows for the voxel-based analysis of DTI data and reduces the effects of local misalignment by projecting all voxels onto the nearest location on a skeleton approximating white matter tract centres. ${ }^{17,18}$ The tbss_1_preproc script in FSL ${ }^{19}$ slightly eroded FA images and zeroed the end slices. All FA images were then aligned to the 
FMRIB58 FA standard space using the tbss_2_reg script. ${ }^{20,21}$ With the tbss_3_postreg script applied to the nonlinear transformations previously generated to each brain, we built the mean FA image and created the mean FA skeleton. Finally, with the tbss_4_prestats script, we thresholded the mean FA skeleton image at the chosen threshold of 0.2 and created the skeletonized FA data for all brains by projecting the FA data onto the mean FA skeleton.

We applied the nonlinear registration transformations derived from the processing of FA maps to MD, AD and RD images by running the script tbss_non_FA. We then projected warped MD, AD and RD data onto the mean FA skeleton.

\section{Statistical analysis}

We performed between-group comparisons of DTI metrics using the permutation-based, nonparametric "randomize" tool in FSL, ${ }^{22}$ with threshold-free cluster enhancement ${ }^{23}$ and family-wise error (FWE) correction across voxels. We set the number of permutations to 5000 and used a threshold of $p<0.1$ to define significant clusters, given the exploratory nature of the study. To account for potentially confounding factors, we included age as a covariate in the analyses. ${ }^{24}$

We performed group comparisons on the skeletonized FA, $\mathrm{MD}, \mathrm{AD}$ and RD images. To define white matter tracts of interest, we used 9 masks (dorsal and ventral cingulum bundle, corticospinal tract, fornix, genu of the corpus callosum, superior cerebellar peduncle, superior corona radiata, SLF and uncinate fasciculus) from the Johns Hopkins University ICBM-DTI-81 atlas $^{25}$ and 3 masks (inferior fronto-occipital fasciculus, inferior longitudinal fasciculus and anterior thalamic radiation) from the Johns Hopkins University white matter tractography atlas. ${ }^{26}$

Because of previously reported asymmetry in white matter microstructure, ${ }^{27}$ we tested the regions of interest separately for each hemisphere. To account for multiple testing across regions of interest, we applied false discovery rate (FDR) correction on the region-of-interest statistics, using a relaxed threshold of $q<0.1$, given the exploratory nature of the study. We also ran exploratory analyses at the whole-brain level on the entire skeletonized FA, MD, AD and RD images. We calculated Cohen $d$ effect sizes ${ }^{28}$ in Matlab R2020b (MathWorks) for clusters with significant between-group differences in DTI metrics. Effect sizes are given as the mean of Cohen $d$ values in each of the significant clusters.

To investigate whether FA was related to PMDD symptom severity in clusters $(k=6)$ that differed between individuals with PMDD and controls, we performed partial correlation analyses, adjusting for age. ${ }^{24}$ We used the Anderson-Darling test to assess normal distribution. We used Pearson and Spearman correlations for normally and non-normally distributed scores, respectively. Because 3 participants did not provide complete symptom ratings, 54 individuals with PMDD were included in these analyses. We used Bonferroni correction for multiple comparisons of the 8 DRSP scores $(p<0.006$, Bonferroni corrected). We conducted correlation analyses, as well as group comparison of demographic and clinical characteristics, using the SPSS version 27 (SPSS Inc., IBM SPSS Statistics) and Matlab R2020b.

\section{Study II: white matter volume}

\section{Participants}

The VBM study was based on 2 neuroimaging data sets collected at the Departments of Obstetrics and Gynecology at Uppsala University (site 1) and the Umeå Centre for Functional Brain Imaging (site 2) in Sweden. In total, we recruited 94 individuals with PMDD (62 from site 1 and 32 from site 2) and 43 controls (11 from site 1 and 32 from site 2) with regular menstrual cycles, as described for study I. Exclusion criteria, confirmation of PMDD diagnosis and monitoring of the menstrual cycle were identical to what has been described for study I.

All procedures were approved by the ethics committees of Uppsala University (Dnr. 2016/184 and 2016/312) and Umeå University (2016-111-31M, 2017-266-32M), and we obtained written informed consent from all participants.

Six participants (5 PMDD, 1 control) were excluded from the analyses because of technical issues $(n=4)$, a brain tumour $(n=1)$ or claustrophobia $(n=1)$, for a total sample of 89 individuals with PMDD and 42 controls (Appendix 1, Figure S1).

\section{MRI acquisition}

All participants underwent $T_{1}$-weighted MRI scanning during the luteal phase of their menstrual cycle.

At site 1, we acquired high-resolution MRI data with a 3.0 T whole-body scanner (Achieva dStream, Philips Medical Systems) equipped with a 32-channel head coil. We acquired the data using a magnetization-prepared rapid gradient echo sequence with the following parameters: repetition time $8.3 \mathrm{~ms}$; echo time $3.8 \mathrm{~ms}$; voxel size $0.94 \times 0.94 \times 1 \mathrm{~mm}^{3}$; matrix $256 \times 256$; flip angle $8^{\circ} ; 220$ transversal slices; acquisition time $3 \mathrm{~min}, 50 \mathrm{sec}$.

At site 2, we acquired high-resolution MRI data with a 3.0 T scanner (Discovery MR750, General Electric) equipped with a 32-channel head coil. We acquired the data using a 3-dimensional fast spoiled gradient echo sequence with the following parameters: repetition time $8.2 \mathrm{~ms}$; echo time $3.2 \mathrm{~ms}$; voxel size $0.48 \times 0.48 \times 1 \mathrm{~mm}^{3}$; matrix $512 \times 512$; flip angle $12^{\circ} ; 176$ transversal slices; acquisition time $8 \mathrm{~min}, 11 \mathrm{~s}$.

\section{Data preprocessing}

We carried out MRI preprocessing in SPM12 (Wellcome Trust Centre for Neuroimaging) implemented in Matlab R2019b (MathWorks). First, all images were manually reoriented using the anterior commissure as origin $(0,0,0)$. Then, using the segment routine in SPM12, the reoriented images were spatially normalized into Montreal Neurological Institute (MNI) space and corrected for intensity variations before being segmented into grey matter, white matter, cerebrospinal fluid, bone, soft tissue and background based on voxel intensities from MNI tissue probability maps. ${ }^{29}$ After segmentation, the pipeline applied a modulation process on the white matter probability maps to compensate for the effects of spatial normalization on volumetric data (the original voxels were projected into their new location in the warped images). Finally, modulated white matter probability maps were smoothed using an $8 \mathrm{~mm}$ full-width at half maximum Gaussian kernel, resulting in a voxel size of $1.5 \times 1.5 \times 1.5 \mathrm{~mm}^{3}$. 
We followed a quality assessment procedure, including visual inspection and automated quality control, using the CAT12 toolbox in SPM12 (http://dbm.neuro.uni-jena.de/ vbm/check-sample-homogeneity) to detect artifacts and anatomic outliers. Based on brain segmentation, the mean ( \pm standard deviation) total white matter volume was $0.41 \pm$ $0.04 \mathrm{~L}$. The mean ( \pm standard deviation) total intracranial volume was $1.51 \pm 0.11 \mathrm{~L}$.

\section{Statistical analysis}

We carried out between-group comparisons of white matter volume using the nonparametric, permutation-based, threshold-free cluster enhancement method in SPM12, using 5000 permutations and a significance threshold of $p_{\mathrm{FWE}}<0.1$ across voxels. We included total intracranial volume, age and site as confounding covariates, because of their potential influence on volumetric measures.

We conducted VBM voxel-wise group comparisons of white matter probability maps within the predefined bilateral regions of interest used in study I. To account for multiple testing across regions of interest, we applied FDR correction, using a relaxed threshold of $q<0.1$, given the exploratory nature of the study. As well, VBM exploratory analysis consisted of a voxel-wise group comparison conducted within a mask of white matter defined by an absolute threshold set at 0.1 . We generated Cohen $d$ effect-size maps in SPM12 using the "transform and threshold SPM maps" module from the CAT12 toolbox. Effect sizes are given as the mean of Cohen $d$ values within a significant cluster.

To investigate whether differences in white matter volume between individuals with PMDD and controls were related to PMDD symptom severity during the cycle in which they were scanned, we performed nonparametric partial correlation analyses between DRSP scores and white matter volume in individuals with PMDD from site 1, for whom DRSP ratings in the scanning month were available $(n=55)$. We used the total DRSP score and the 8 items related to the core affective PMDD symptoms tested in study I, and we considered total intracranial volume and age as confounding covariates. We carried out these analyses in SPSS version 26 (SPSS Inc.), and set the significance threshold at $p<$ 0.05 , corrected for multiple testing (8 DRSP scores: $p<0.006$, Bonferroni corrected).

\section{Results}

\section{Descriptive characteristics}

The demographic and psychometric characteristics of participants from both studies are presented in Table 1.

Across both studies, individuals with PMDD were slightly older than controls. In the DTI study, individuals with PMDD had higher progesterone levels than controls; in the VBM study, individuals with PMDD reported a psychiatric history more often than controls. Individuals with PMDD and controls did not differ in terms of body mass index, total intracranial volume, menstrual cycle length or estradiol levels.

\section{Between-group differences in white matter microstructure}

Within a priori-defined regions of interest, with effect sizes indicating strong differences, we found higher FA in individuals with PMDD versus controls in the left dorsal cingulum bundle $(d=0.71)$, the right dorsal cingulum bundle $(d=0.83)$, the left genu of the corpus callosum $(d=0.87)$, the right SLF $(d=0.75)$, the left uncinate fasciculus $(d=0.69)$ and the right

Table 1: Participant characteristics

\begin{tabular}{|c|c|c|c|c|}
\hline \multirow[b]{2}{*}{ Characteristic } & \multicolumn{2}{|c|}{ Diffusion tensor imaging (study I)* } & \multicolumn{2}{|c|}{ Voxel-based morphometry (study II)* } \\
\hline & Controls $(n=10)$ & $\operatorname{PMDD}(n=57)$ & Controls $(n=42)$ & $\operatorname{PMDD}(n=89)$ \\
\hline Age, yr & $30.30 \pm 6.70$ & $35.14 \pm 6.31 \S$ & $28.00 \pm 6.00$ & $33.00 \pm 7.00 \S$ \\
\hline Body mass index, $\mathrm{kg} / \mathrm{m}^{2}$ & $22.94 \pm 3.11$ & $24.45 \pm 4.34$ & $23.80 \pm 3.80$ & $24.10 \pm 4.00$ \\
\hline Total intracranial volume, $\mathrm{L}$ & $1.47 \pm 0.10$ & $1.47 \pm 0.10$ & $1.52 \pm 0.11$ & $1.50 \pm 0.10$ \\
\hline Menstrual cycle length, $d$ & $27 \pm 2$ & $28 \pm 2$ & $28 \pm 2$ & $28 \pm 2$ \\
\hline Psychiatric history† & $4(40.0)$ & $24(42.1)$ & $7(16.6)$ & $34(39.1) \emptyset$ \\
\hline Depressive disorder & $3(75.0)$ & $18(75)$ & $6(85.7)$ & $27(79.4)$ \\
\hline Anxiety disorder & $1(25.0)$ & $6(25)$ & $1(14.3)$ & $6(17.6)$ \\
\hline Eating disorder & $0(0)$ & $3(12.5)$ & $0(0)$ & $4(11.8)$ \\
\hline Estradiol, pmol/Lł & $286.39 \pm 210.13$ & $414.00 \pm 251.37$ & $434.50 \pm 47.40$ & $411.10 \pm 239.80$ \\
\hline Progesterone, nmol/Lł & $9.26 \pm 15.06$ & $21.25 \pm 16.06 \S$ & $18.70 \pm 15.00$ & $21.90 \pm 15.30$ \\
\hline DRSP, total score§ & NA & $61.67 \pm 18.39$ & NA & $61.80 \pm 18.20$ \\
\hline \multicolumn{5}{|c|}{$\begin{array}{l}\text { DRSP = Daily Record of Severity of Problems; NA = not applicable; PMDD = premenstrual dysphoric disorder. } \\
\text { *Values are mean } \pm \text { standard deviation or } n(\%) \text {. Differences between groups were assessed using the Mann-Whitney test for continuous variables and the Fisher exact test for } \\
\text { categorical variables. } \\
\text { †The percentage of participants who reported a psychiatric history is based on the full sample; participants could report more than } 1 \text { disorder. Percentages for depressive, anxiety and } \\
\text { eating disorders is based on the number of participants who reported a psychiatric history. In study II, } 2 \text { participants in the PMDD group did not provide information about previous } \\
\text { psychiatric disorders. } \\
\text { fHormone data were available for a subset of } 9 \text { controls and } 55 \text { individuals with PMDD in the diffusion tensor imaging study, and } 39 \text { controls and } 85 \text { individuals with PMDD in the voxel- } \\
\text { based morphometry study. } \\
\text { §DRSP scores were available for a subset of } 54 \text { individuals with PMDD in the diffusion tensor imaging study, and } 55 \text { in the voxel-based morphometry study. } \\
\text { TSignificant group difference at } p<0.05 \text {. }\end{array}$} \\
\hline
\end{tabular}


uncinate fasciculus ( $d=0.67$; Figure $1 \mathrm{~A}$ and B and Table 2). Of these, the cluster in the left uncinate fasciculus survived FDR correction. At the whole-brain level, we noted no additional significant differences in FA between groups.

For diffusivity metrics, we found no significant difference in RD between groups. As illustrated in Appendix 1, Figures S2 and S3, MD and AD were higher in individuals with PMDD than controls in different regions of interest (Appendix 1, Table S1).

\section{Between-group differences in white matter volume}

After FDR correction across the tested regions of interest, we found that individuals with PMDD had greater white matter volume in the right uncinate fasciculus compared to controls (Figure 2 and Table 2). Effect sizes in the significant cluster indicated moderate differences $(d=0.40)$. At the whole-brain level, and in the other regions of interest, we found no differences in white matter volume between groups.
A

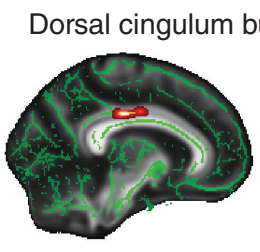

Genu of the corpus callosum
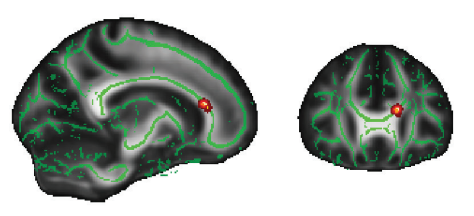

Superior longitudinal fasciculus
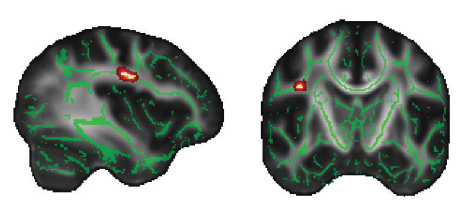

Uncinate fasciculus
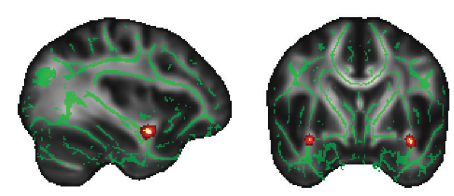

PMDD $>$ control, $p_{\text {FWE }}<0.1$

\section{B}

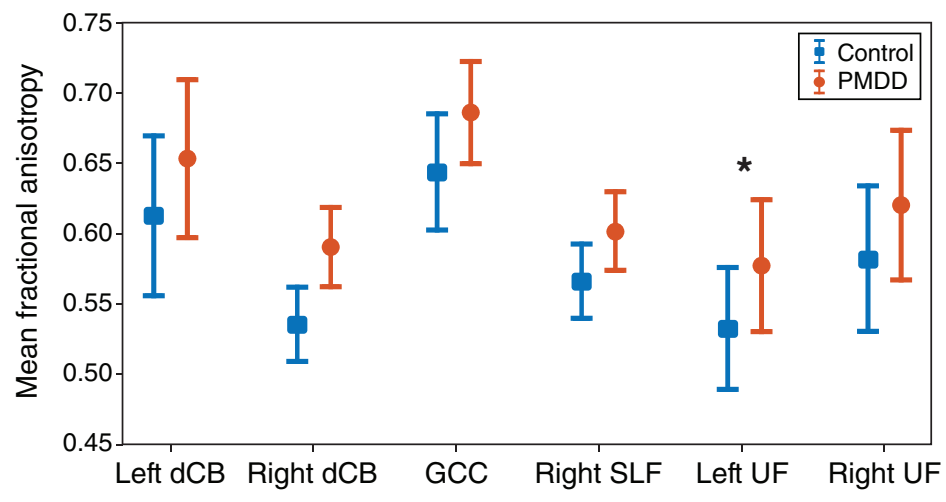

C

Right SLF

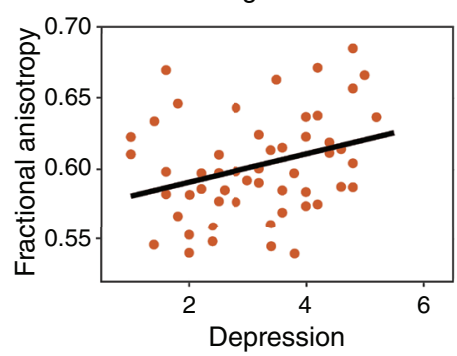

Right SLF

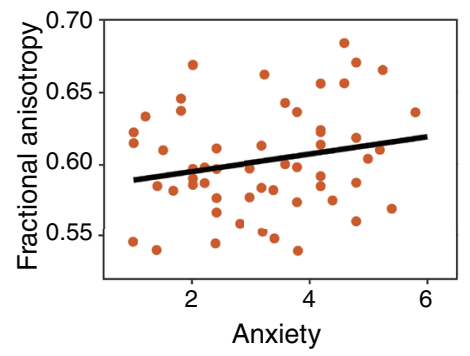

Figure 1: Fractional anisotropy results in regions of interest. (A) Results from tract-based spatial statistics showing between-group differences in fractional anisotropy. Depicted in red-yellow are the 6 clusters in which we found greater fractional anisotropy in individuals with premenstrual dysphoric disorder compared to controls. The clusters are overlaid onto the mean fractional anisotropy skeleton (green) and displayed in 3-dimensional view at their peak Montreal Neurological Institute coordinates, in the standard radiology orientation (left-right flip). For visualization, results were thickened using the tbss_fill command in FSL. Results are visualized at a threshold of $p_{\mathrm{FWE}}<0.1$, corrected for multiple comparisons across voxels with threshold-free cluster enhancement. (B) Between-group differences in fractional anisotropy in significant or trend clusters at $p<0.1$ (left dorsal cingulum bundle $p=0.031$; right dorsal cingulum bundle $p=0.070$; genu of the corpus callosum $p=$ 0.095 ; right superior longitudinal fasciculus $p=0.031$; left uncinate fasciculus $p=0.024$; right uncinate fasciculus $p=0.081$ ). Error bars show 1 standard deviation across participants. * False discovery rate corrected across regions of interest at $q<0.1$. (C) Correlation between scores on the Daily Record of Severity of Problems scale and fractional anisotropy values in clusters with a significant group difference at $p<0.1$. We found positive associations between fractional anisotropy values from the right superior longitudinal fasciculus and Daily Record of Severity of Problems scores for depression $(r=0.350, p=0.010)$ and for anxiety at a trend level $(r=0.262, p=0.058)$. dCB $=$ dorsal cingulum bundle; FWE = family-wise error; GCC = genu of the corpus callosum; PMDD = premenstrual dysphoric disorder; SLF = superior longitudinal fasciculus; UF = uncinate fasciculus. 
Table 2: Between-group differences in fractional anisotropy and white matter volume in regions of interest*

\begin{tabular}{|c|c|c|c|c|c|c|}
\hline \multirow[b]{2}{*}{ Tract of interest } & \multirow{2}{*}{$\begin{array}{l}\text { Cluster size } \\
\text { voxels }\end{array}$} & \multicolumn{2}{|c|}{ Mean value $\pm S D$} & \multirow[b]{2}{*}{ TFCE $p$ value } & \multirow[b]{2}{*}{ Cohen $d$} & \multirow{2}{*}{$\begin{array}{c}\text { MNI coordinates } \\
\text { of peak voxel, } \\
x, y, z\end{array}$} \\
\hline & & PMDD & Controls & & & \\
\hline \multicolumn{7}{|l|}{ Fractional anisotropy, PMDD > controls } \\
\hline Left dorsal cingulum bundle & 57 & $0.65 \pm 0.06$ & $0.61 \pm 0.06$ & 0.031 & 0.71 & $-9,-21,33$ \\
\hline Right dorsal cingulum bundle & 26 & $0.59 \pm 0.03$ & $0.54 \pm 0.03$ & 0.070 & 0.83 & $9,9,31$ \\
\hline Genu of the corpus callosum & 15 & $0.69 \pm 0.04$ & $0.64 \pm 0.04$ & 0.095 & 0.87 & $-14,30,16$ \\
\hline Right superior longitudinal fasciculus & 115 & $0.60 \pm 0.03$ & $0.57 \pm 0.03$ & 0.031 & 0.75 & $39,-10,29$ \\
\hline Left uncinate fasciculus & 30 & $0.58 \pm 0.05$ & $0.53 \pm 0.04$ & $0.024 \dagger$ & 0.69 & $-35,-1,-13$ \\
\hline Right uncinate fasciculus & 12 & $0.62 \pm 0.05$ & $0.58 \pm 0.05$ & 0.081 & 0.67 & $34,2,-12$ \\
\hline \multicolumn{7}{|l|}{ White matter volume, PMDD > controls } \\
\hline Right uncinate fasciculus & 110 & $0.24 \pm 0.05$ & $0.22 \pm 0.05$ & $0.010 \dagger$ & 0.40 & $36,0,-14$ \\
\hline $\begin{array}{l}\text { MNI = Montreal Neurological Institute; PMDD } \\
{ }^{*} \text { Threshold-free cluster enhancement voxel-w } \\
\text { Mean raw values of fractional anisotropy and } \\
\text { comparisons using the family-wise error rate } \\
t q<0.1 \text {, corrected for false discovery rate ac }\end{array}$ & $\begin{array}{l}\text { nenstrual dysphor } \\
\text { mparisons of fract } \\
\text { matter volume we } \\
0.1 \text { across voxels. } \\
\text { egions of interest. }\end{array}$ & $\begin{array}{l}\text { rder; SD = stan } \\
\text { nisotropy and } \\
\text { acted from signi }\end{array}$ & $\begin{array}{l}\text { viation; TFCE = } \\
\text { tter volume betv } \\
\text { lusters and are }\end{array}$ & $\begin{array}{l}\text { nold-free cluster en } \\
\text { ndividuals with PM } \\
\text { ted for each group }\end{array}$ & $\begin{array}{l}\text { nent. } \\
\text { healthy con } \\
\text { ts are correc }\end{array}$ & $\begin{array}{l}\text { tracts of interest. } \\
\text { multiple }\end{array}$ \\
\hline
\end{tabular}
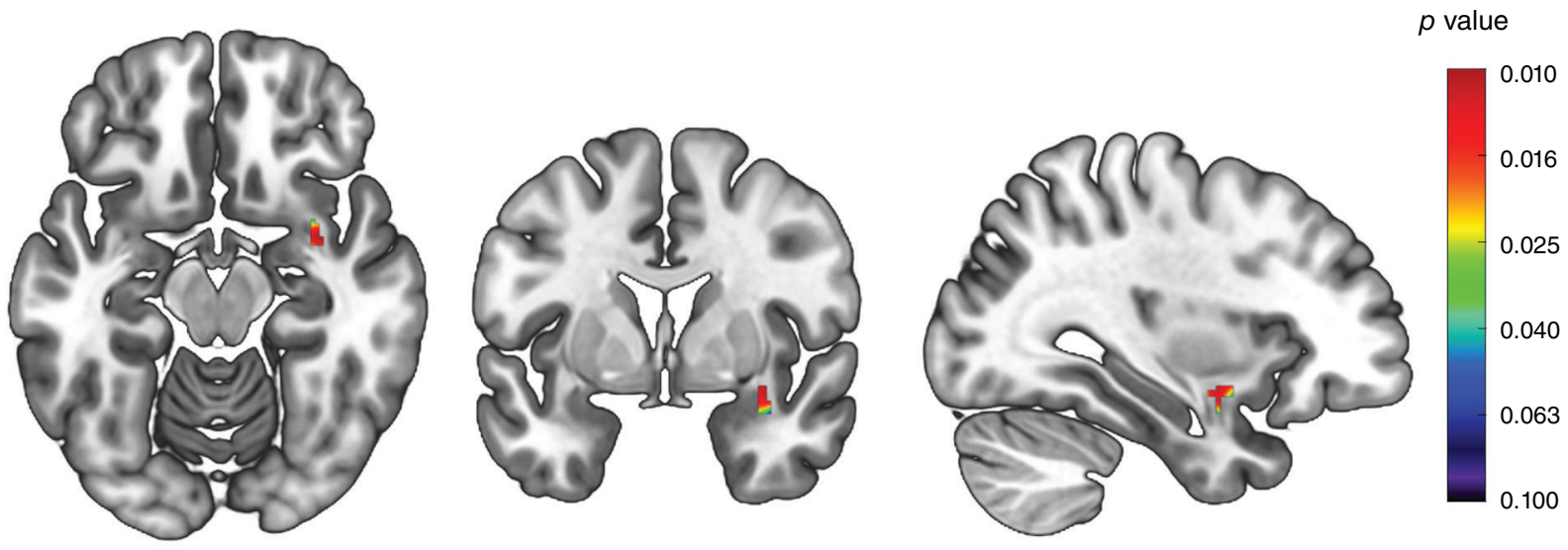

Figure 2: Between-group differences in white matter volume in the uncinate fasciculus. Threshold-free cluster enhancement voxel-wise comparisons showing higher white matter volume in individuals with premenstrual dysphoric disorder compared to healthy controls. The significant cluster (peak at $x, y, z=36,0,-14 ; k=110$ voxels; $p_{\text {FWE }}=0.010$; threshold-free cluster enhancement) has been overlaid onto a Montreal Neurological Institute standard template. The colour bar indicates $p$ values after FWE correction across voxels. FWE $=$ family-wise error.

\section{Correlations between symptom severity and white matter metrics}

In individuals with PMDD, FA values were not correlated with total DRSP scores or individual core affective symptoms after correction for multiple testing. However, at the uncorrected level, we found trend-level positive associations between FA values in the right SLF and individual DRSP items such as depression $(r=0.35, p=0.010)$ and anxiety $(r=0.26$, $p=0.058$; Figure 1C). White matter volume in the differential cluster of the right uncinate fasciculus did not correlate with symptom severity.

\section{Discussion}

The present studies sought to investigate whether white matter structure is altered in individuals with PMDD compared to healthy controls, using DTI and VBM analyses. We compared DTI metrics and white matter volumes in individuals with PMDD and healthy controls scanned during the symptomatic phase of the menstrual cycle, in 2 neuroimaging studies. An additional purpose of these studies was to determine whether differential white matter metrics were associated with symptom severity in individuals with PMDD. 
Our findings defined, for the first time, the brain structural correlates of PMDD using tract-based spatial statistics and DTI metrics of white matter microstructure, as well as VBM metrics of white matter volume. We observed greater FA in individuals with PMDD compared to controls in the bilateral dorsal cingulum bundle, the bilateral uncinate fasciculus, the left genu of the corpus callosum and the right SLF. Interestingly, AD differed between individuals with PMDD and controls in the same direction and in regions that overlapped with the FA findings, indicating that differences in FA in these regions were likely driven by differences in AD. We detected greater white matter volume in individuals with PMDD than controls in the right uncinate fasciculus. We also reported trend-level positive associations between FA values in the right SLF and symptom severity in individuals with PMDD, reflecting the relationship between white matter structure and premenstrual mood. Taken together, these results are of relevance to PMDD, because these tracts bridge cortical and subcortical areas, particularly corticolimbic connections, and thus could align with the hypothesis of altered top-down control in PMDD. ${ }^{30}$

Functional neuroimaging studies suggest an impaired topdown process in the affective networks in PMDD. ${ }^{4}$ This could relate to reduced white matter integrity in tracts that connect cortical control areas and subcortical regions of the limbic system in PMDD. Such a relationship would be in line with DTI studies in psychiatric disorders, pointing to lower FA in patients with schizophrenia, mood disorders and autism. ${ }^{31-33}$ Conversely, our findings of higher white matter integrity in the brains of individuals with PMDD suggest that compensatory neuroadaptive mechanisms might counteract the dysregulation of corticolimbic regions. Indeed, the etiology of this hormone-dependent disorder is likely to diverge from the etiology of other psychiatric disorders, in light of the cyclical aspect of PMDD symptomatology. Moreover, although the present DTI findings were limited by the small size of the control group, the VBM results obtained from a larger group of individuals with PMDD and controls followed the same direction, as did the correlations with symptom severity, supporting the validity of these findings.

We detected higher FA and white matter volume in individuals with PMDD in the uncinate fasciculus (significant after correction for multiple testing), a white matter tract that connects the prefrontal cortex and the anteromedial temporal lobe, ${ }^{34}$ including connections between regions of the limbic system, such as the amygdala and the orbitofrontal cortex. The amygdala is one of the key regions hypothesized to play a role in the pathophysiology of PMDD, in the context of an altered top-down inhibitory process. ${ }^{4}$ For instance, compared to controls, individuals with PMDD show enhanced reactivity to social stimuli in the amygdala during the symptomatic luteal phase. ${ }^{35}$ As well, greater reactivity to negative emotional stimuli in the amygdala and the orbitofrontal cortex has been reported in individuals with PMDD in the late luteal phase. ${ }^{36}$ Therefore, the differential FA and white matter volume we found in the uncinate fasciculus in individuals with PMDD compared with controls may relate to the functional variations reported in the amygdala and orbitofrontal cortex in individuals with PMDD. ${ }^{4}$
Individuals with PMDD also had higher FA in the midcingulate subdivision of the dorsal cingulum bundle compared to controls. The cingulum is the most important white matter structure in the limbic system, linking the cingulate gyrus to subcortical regions, as well as to frontal, parietal and medial temporal areas. ${ }^{37}$ It plays an important role in emotion regulation, attention, motivation and working memory. This finding is of relevance to PMDD, especially considering previous functional MRI studies on emotion processing. ${ }^{35,38}$ Specifically, lower brain activation during emotion processing was found in the anterior cingulate cortex in individuals with PMDD, independent of menstrual cycle phase ${ }^{38}$ or solely in the luteal phase..$^{35}$ Because altered function of the corticolimbic system is one of the core findings of neuroimaging studies on PMDD ${ }^{4}$ a greater microstructural coherence of the white matter tract interconnecting the cingulate gyrus with the frontal and subcortical regions may represent compensatory mechanisms resulting from poor top-down communication between cognitive and emotional circuits. This could also relate to differential resting-state activity in the default mode network, which remains understudied in PMDD ${ }^{39}$ and whose key nodes largely overlap with the termination regions of the cingulum. ${ }^{40}$

The right SLF was also characterized by higher FA measures in component II terminating in the dorsolateral prefrontal corte ${ }^{41}$ in individuals with PMDD. This finding was in line with those of a study that provided evidence for increased FA in bipolar disorder in the right SLF. ${ }^{42}$ However, it contrasted with the results of a meta-analysis that showed that the majority of studies of FA in emotional disorders reported reduced FA in the left SLF. The SLF connects the prefrontal cortex in each cerebral hemisphere with the parietal lobe. ${ }^{43}$ Interestingly, several reports of variations in the function of the prefrontal cortex and parietal regions have been made in PMDD, although the direction of effect seems to depend on the specific paradigms used in each functional MRI study. ${ }^{4}$ In addition, evidence from resting-state functional MRI points to stronger connectivity involving the executive control network, which links the dorsolateral prefrontal cortex and parietal cortex, ${ }^{44}$ in individuals with PMDD. ${ }^{45}$ Hence, the greater FA found in the right SLF in individuals with PMDD in the present study may relate to stronger functional connectivity, and to differential brain reactivity previously reported in brain networks involving frontal and parietal regions in individuals with PMDD. In line with such findings, we found a positive correlation at a trend level between FA values in the right SLF and the severity of anxiety and depression, 2 key symptoms experienced by individuals with PMDD.

Furthermore, the genu of the corpus callosum was among the areas highlighted by the DTI findings. The corpus callosum connects the left and right cerebral hemispheres and is involved in interhemispheric communication. ${ }^{46}$ The genu consists of a high density of thin fibres that cross over to form the forceps minor, which connects the homologous medial and lateral prefrontal associative areas. ${ }^{47}$ Although there is no consensus on the specific functions of this callosal subregion, it is thought to be relevant to emotion regulation. Neuroimaging studies support this hypothesis, showing a positive association between emotion regulation abilities and FA in 
the forceps minor in healthy participants. ${ }^{48}$ In addition, across emotional disorders, reduced FA in the forceps minor and genu of the corpus callosum characterizes patients compared to controls, ${ }^{6}$ although it seems to be of particular relevance to bipolar disorder. ${ }^{6,49,50}$ In individuals with PMDD, differential reactivity of the medial and dorsolateral prefrontal areas to emotional tasks has been reported, ${ }^{4}$ a finding that could relate to the higher FA in the genu of the corpus callosum we found in individuals with PMDD compared to controls.

\section{Limitations}

Although DTI metrics provide us with unique insights into white matter microstructure, variations in FA and diffusivity metrics should be interpreted with caution. Commonly interpreted as a marker of white matter integrity, FA is under the influence of multiple microstructural features that cannot be disentangled at the voxel level (e.g., degree of myelination, internal axonal structure, axon packing, water content and orientation of large macromolecules and membranes in the tissues). ${ }^{51}$ Moreover, although the degree of myelination correlates with FA, it is not possible to disentangle the effects of axon count and myelin while interpreting FA changes, because they are strongly interrelated. ${ }^{52}$ As for the diffusivity metrics $\mathrm{AD}$ and $\mathrm{RD}$, which contribute to define FA, interpretation in terms of underlying biophysical properties is also challenging. ${ }^{52}$ Animal experiments have suggested that astrocytes, myelin and oligodendrocytes make the greatest contribution to any white matter voxel measurements in human MRIs. ${ }^{53}$ With respect to white matter volume measures derived from $T_{1}$-weighted images, a postmortem quantitative MRI study showed that they are presumably dependent on myelin content. ${ }^{54}$ Therefore, the greater FA and white matter volume found in individuals with PMDD in the present study was most likely to reflect more numerous, myelinated and coherently oriented white matter fibres.

Combining DTI and VBM analyses of white matter is likely to provide complementary information about white matter structure. The direction of effect in the uncinate fasciculus was consistent across imaging modalities in the present study. This contributed to overcoming the limitation of small sample size in study I, which was underpowered to detect moderate effect sizes. ${ }^{55}$

One possible consequence of the small sample was the lower progesterone concentrations found in the control group compared to the PMDD group in the DTI study (study I). Indeed, the reduced variability in menstrual cycle day at scanning and therefore in progesterone concentrations imparted by the small control group in study I could explain this difference.

The exploratory nature of this study should be noted when considering the number of tracts of interest investigated, because only the left uncinate fasciculus remained significant after FDR correction of the DTI findings. Finally, the use of tract-based spatial statistics and threshold-free cluster enhancement provided improved sensitivity compared to traditional voxel-level approaches. ${ }^{18,23}$

\section{Conclusion}

The present findings provide the first evidence of differential cerebral white matter structure associated with PMDD. These findings suggest that structural modifications in white matter tracts that ensure communication between cortical and subcortical limbic and paralimbic regions may be involved in the pathophysiological mechanisms of PMDD. This primary evidence of white matter variations in PMDD calls for independent replication and further investigation into the structure of the specific networks involved in the symptomatology of this mood disorder.

Acknowledgements: Computation of the permutation tests in TBSS was enabled by resources in project sens 2021510 provided by the Swedish National Infrastructure for Computing at UPPMAX, partially funded by the Swedish Research Council through grant agreement no. 2018-05973. The authors would also like to thank the research nurses at the Uppsala University Hospital MR centre and Department of Women's and Children's Health of Uppsala University for their participation in data collection.

Affiliations: From the Department of Neuroscience, Science for Life Laboratory, Uppsala University, Sweden (Gu, Dubol, Comasco); the Department of Clinical Sciences, Umeå University, Umeå, Sweden (Stiernman, Bixo); the Department of Surgical Sciences, Neuroradiology, Uppsala University, Sweden (Wikström); the Department of Psychiatry and Psychotherapy, Medical University of Vienna, Austria (Hahn, Lanzenberger); the Department of Psychiatry, University of Colorado School of Medicine, USA (Epperson); the Department of Women's and Children's Health, Uppsala University, Sweden (Sundström-Poromaa).

Funding: This study was supported by the EU FP7-People-Cofund (INCA 600398), the Swedish Research Council (2015-00495; 201601439; 2020-01801), the Swedish Society of Medicine (SLS-573171, SLS597211, SLS-789101) and the Swedish Brain Foundation (2020-0255).

Competing interests: $R$. Lanzenberger received support from Siemens Healthcare for clinical research using PET/MR, conference speaker honoraria and travel grants within the last 3 years from Bruker BioSpin MR and Heel, and he has been a shareholder of the start-up company BM Health GmbH since 2019. C.N. Epperson has received research grant support from Sage Therapeutics; he has served on an advisory board for Sage Therapeutics and has received honoraria and travel funding from Sage Therapeutics. I. SundströmPoromaa was supported by a grant from the Swedish Research Council (2020-01801) and the Brain Foundation (F2020-0255), has served occasionally on advisory boards for Gedeon Richter and received honoraria as an invited speaker for Bayer Health Care, Gedeon Richter, Peptonics, Shire/Takeda, and Sandoz. E. Comasco receives funds from SciLifeLab. No other competing interests declared.

Contributors: I. Sundström-Poromaa and E. Comasco designed the study. J. Wikström and M. Bixo acquired the data, which X. Gu, M. Dubol, L. Stiernman, A. Hahn, R. Lanzenberger and N. Epperson analyzed. X. Gu, M. Dubol and E. Comasco wrote the article, which L. Stiernman, J. Wikström, A. Hahn, R. Lanzenberger, N. Epperson, M. Bixo and I. Sundström-Poromaa reviewed. All authors approved the final version to be published and can certify that no other individuals not listed as authors have made substantial contributions to the paper.

Data availability: Data are available upon request.

Content licence: This is an Open Access article distributed in accordance with the terms of the Creative Commons Attribution (CC BYNC-ND 4.0) licence, which permits use, distribution and reproduction in any medium, provided that the original publication is properly cited, the use is noncommercial (i.e., research or educational use), and no modifications or adaptations are made. See: https://creativecommons.org/licenses/by-nc-nd/4.0/ 


\section{References}

1. Halbreich U, Borenstein J, Pearlstein T, et al. The prevalence, impairment, impact, and burden of premenstrual dysphoric disorder (PMS/PMDD). Psychoneuroendocrinology 2003;28:1-23.

2. American Psychiatric Association. Diagnostic and statistical manual of mental disorders. Fifth edition. Arlington (VA): American Psychiatric Association Publishing; 2013.

3. Epperson CN, Steiner M, Hartlage SA, et al. Premenstrual dysphoric disorder: evidence for a new category for DSM-5. Am J Psychiatry 2012;169:465-75.

4. Dubol M, Epperson CN, Lanzenberger R, et al. Neuroimaging premenstrual dysphoric disorder: a systematic and critical review. Front Neuroendocrinol 2020;57:100838.

5. Le Bihan D. Looking into the functional architecture of the brain with diffusion MRI. Nat Rev Neurosci 2003;4:469-80.

6. Jenkins LM, Barba A, Campbell M, et al. Shared white matter alterations across emotional disorders: a voxel-based meta-analysis of fractional anisotropy. Neuroimage Clin 2016;12:1022-34.

7. Dillon DG, Gonenc A, Belleau E, et al. Depression is associated with dimensional and categorical effects on white matter pathways. Depress Anxiety 2018;35:440-7.

8. Olson EA, Cui J, Fukunaga R, et al. Disruption of white matter structural integrity and connectivity in posttraumatic stress disorder: a TBSS and tractography study. Depress Anxiety 2017;34:437-45.

9. Dubol M, Epperson CN, Sacher J, et al. Neuroimaging the menstrual cycle: a multimodal systematic review. Front Neuroendocrinol 2021;60:100878.

10. Barth C, Steele CJ, Mueller K, et al. In-vivo dynamics of the human hippocampus across the menstrual cycle. Sci Rep 2016;6:32833.

11. Endicott J, Nee J, Harrison W. Daily record of severity of problems (DRSP): reliability and validity. Arch Womens Ment Health 2006;9:41-9.

12. Eisenlohr-Moul TA, Girdler SS, Schmalenberger KM, et al. Toward the reliable diagnosis of DSM-5 premenstrual dysphoric disorder: the Carolina Premenstrual Assessment Scoring System (C-PASS). Am J Psychiatry 2017;174:51-9.

13. Sheehan DV, Lecrubier $\mathrm{Y}$, Sheehan $\mathrm{KH}$, et al. The MiniInternational Neuropsychiatric Interview (MINI): the development and validation of a structured diagnostic psychiatric interview for DSM-IV and ICD-10. J Clin Psychiatry 1998;59:22-33.

14. Jenkinson M, Smith S. A global optimisation method for robust affine registration of brain images. Med Image Anal 2001;5:143-56.

15. Jenkinson M, Bannister P, Brady M, et al. Improved optimization for the robust and accurate linear registration and motion correction of brain images. Neuroimage 2002;17:825-41.

16. Smith SM. Fast robust automated brain extraction. Hum Brain Mapp 2002;17:143-55.

17. Bach M, Laun FB, Leemans A, et al. Methodological considerations on tract-based spatial statistics (TBSS). Neuroimage 2014;100:358-69.

18. Smith SM, Jenkinson M, Johansen-Berg H, et al. Tract-based spatial statistics: voxelwise analysis of multi-subject diffusion data. Neuroimage 2006;31:1487-505.

19. Jenkinson M, Beckmann CF, Behrens TE, et al. FSL. Neuroimage 2012;62:782-90.

20. Andersson J, Jenkinson M, Smith S. Non-linear optimisation. Oxford: FMRIB, University of Oxford; 2007. Report no TR07JA1.

21. Andersson J, Jenkinson M, Smith S. Non-linear registration. Oxford: FMRIB, University of Oxford; 2007. Report no TR07JA2.

22. Winkler AM, Ridgway GR, Webster MA, et al. Permutation inference for the general linear model. Neuroimage 2014;92:381-97.

23. Smith SM, Nichols T. Threshold-free cluster enhancement: addressing problems of smoothing, threshold dependence and localisation in cluster inference. Neuroimage 2009;44:83-98.
24. Sexton $\mathrm{CE}$, Walhovd KB, Storsve AB, et al. Accelerated changes in white matter microstructure during aging: a longitudinal diffusion tensor imaging study. J Neurosci 2014;34:15425-36.

25. Mori S, Oishi K, Jiang H, et al. Stereotaxic white matter atlas based on diffusion tensor imaging in an ICBM template. Neuroimage 2008;40:570-82

26. Hua K, Zhang J, Wakana S, et al. Tract probability maps in stereotaxic spaces: analyses of white matter anatomy and tract-specific quantification. Neuroimage 2008;39:336-47.

27. Honnedevasthana Arun A, Connelly A, Smith RE, et al. Characterisation of white matter asymmetries in the healthy human brain using diffusion MRI fixel-based analysis. Neuroimage 2021;225:117505.

28. Cohen J. Statistical power analysis for the behavioral sciences. Cambridge (MA): Academic Press; 2013.

29. Ashburner J, Friston KJ. Voxel-based morphometry - the methods. Neuroimage 2000;11:805-21.

30. Comasco E, Sundström-Poromaa I. Neuroimaging the menstrual cycle and premenstrual dysphoric disorder. Curr Psychiatry Rep 2015;17:77.

31. Koshiyama D, Fukunaga M, Okada N, et al. White matter microstructural alterations across four major psychiatric disorders: megaanalysis study in 2937 individuals. Mol Psychiatry 2020;25:883-95.

32. Podwalski P, Szczygiel K, Tyburski E, et al. Magnetic resonance diffusion tensor imaging in psychiatry: a narrative review of its potential role in diagnosis. Pharmacol Rep 2021;73:43-56.

33. White T, Nelson M, Lim KO. Diffusion tensor imaging in psychiatric disorders. Top Magn Reson Imaging 2008;19:97-109.

34. Bhatia K, Henderson L, Yim M, et al. Diffusion tensor imaging investigation of uncinate fasciculus anatomy in healthy controls: description of a subgenual stem. Neuropsychobiology 2017;75:132-40.

35. Gingnell M, Ahlstedt V, Bannbers E, et al. Social stimulation and corticolimbic reactivity in premenstrual dysphoric disorder: a preliminary study. Biol Mood Anxiety Disord 2014;4:3.

36. Protopopescu X, Tuescher O, Pan H, et al. Toward a functional neuroanatomy of premenstrual dysphoric disorder. J Affect Disord 2008;108:87-94.

37. Bubb EJ. The cingulum bundle: anatomy, function, and dysfunction. Neurosci Biobehav Rev 2018;92:104-127.

38. Comasco E, Hahn A, Ganger S, et al. Emotional fronto-cingulate cortex activation and brain derived neurotrophic factor polymorphism in premenstrual dysphoric disorder. Hum Brain Mapp 2014;35:4450-8.

39. Syan SK, Minuzzi L, Smith M, et al. Brain structure and function in women with comorbid bipolar and premenstrual dysphoric disorder. Front Psychiatry 2018;8:301.

40. van den Heuvel M, Mandl R, Luigjes J, et al. Microstructural organization of the cingulum tract and the level of default mode functional connectivity. J Neurosci 2008;28:10844-51.

41. Makris N, Kennedy DN, McInerney S, et al. Segmentation of subcomponents within the superior longitudinal fascicle in humans: a quantitative, in vivo, DT-MRI study. Cereb Cortex 2005;15:854-69.

42. Versace A, Almeida JRC, Hassel S, et al. Elevated left and reduced right orbitomedial prefrontal fractional anisotropy in adults with bipolar disorder revealed by tract-based spatial statistics. Arch Gen Psychiatry 2008;65:1041.

43. Schmahmann JD, Smith EE, Eichler FS, et al. Cerebral white matter: neuroanatomy, clinical neurology, and neurobehavioral correlates. Ann N Y Acad Sci 2008;1142:266.

44. Seeley WW, Menon V, Schatzberg AF, et al. Dissociable intrinsic connectivity networks for salience processing and executive control. J Neurosci 2007;27:2349-56.

45. Petersen N, Ghahremani DG, Rapkin AJ, et al. Resting-state functional connectivity in women with PMDD. Transl Psychiatry 2019;9:339. 
46. Gazzaniga MS. Cerebral specialization and interhemispheric communication: does the corpus callosum enable the human condition? Brain 2000;123:1293-326.

47. Raybaud C. The corpus callosum, the other great forebrain commissures, and the septum pellucidum: anatomy, development, and malformation. Neuroradiology 2010;52:447-77.

48. Pisner DA, Smith R, Alkozei A, et al. Highways of the emotional intellect: white matter microstructural correlates of an ability-based measure of emotional intelligence. Soc Neurosci 2017;12:253-67.

49. Cyprien F, de Champfleur NM, Deverdun J, et al. Corpus callosum integrity is affected by mood disorders and also by the suicide attempt history: a diffusion tensor imaging study. J Affect Disord 2016;206:115-24.

50. Zhang R, Jiang $X$, Chang $M$, et al. White matter abnormalities of corpus callosum in patients with bipolar disorder and suicidal ideation. Ann Gen Psychiatry 2019;18:20.

51. Beaulieu C. The basis of anisotropic water diffusion in the nervous system - a technical review. NMR Biomed 2002;15:435-55.

52. Curran KM, Emsell L, Leemans A. Quantitative DTI measures. In: Diffusion tensor imaging. Berlin: Springer; 2016: 65-87.

53. Walhovd KB, Johansen-Berg H, Karadottir RT. Unraveling the secrets of white matter - bridging the gap between cellular, animal and human imaging studies. Neuroscience 2014;276:2-13.

54. Stüber C, Morawski M, Schäfer A, et al. Myelin and iron concentration in the human brain: a quantitative study of MRI contrast. Neuroimage 2014;93:95-106.

55. Faul F, Erdfelder E, Buchner A, et al. Statistical power analyses using $\mathrm{G}^{*}$ Power 3.1: tests for correlation and regression analyses. Behav Res Methods 2009;41:1149-60. 